\title{
Will the COVID-19 Pandemic Revolutionize the Psychiatric Healthcare System?
}

\author{
Karina Badura Brzoza* and Patryk Główczyński \\ Department of Psychiatry in Tarnowskie Góry, Medical University of Silesia in Katowice, Poland
}

*Corresponding author: Karina Badura Brzoza, Department of Psychiatry in Tarnowskie Góry, The Faculty of Medical Sciences in Zabrze, Medical University of Silesia in Katowice, Poland

To Cite This Article: Karina Badura Brzoza. Will the COVID-19 Pandemic Revolutionize the Psychiatric Healthcare System?. 2020 - 10(2). AJBSR. MS.ID.001498.

DOI: 10.34297/AJBSR.2020.10.001498

Received: 鮆 September 06, 2020; Published: 眥 September 11, 2020

\begin{abstract}
The general definition determines telemedicine as a remote medical care which, apart from the process of diagnosis and treatment itself, also emphasizes the importance of medical education of patients [1]. Another, more precise definition says that telemedicine is a form of exchange of medical information between two partners, using telecommunications, to improve the patient's health [2]. The development of telemedicine dates to the 1960, where the transfer of medical data via various communication systems was mainly used by the military services (NASA). Subsequently, this system began to develop intensively, mainly in countries where access to health care was limited, mainly due to the significant distance between patients and doctors (Australia, USA, Scandinavia)[3]. In recent years, more and more specialties have started to use forms of telemedicine, one of them being psychiatry. Originally, telepsychiatry was intended to be a helpful method in the treatment of patients living in rural areas or in regions with insufficient public transport, where patients had problems with reaching a specialist. The useage of telepsychiatry for emergency interventions was also promoted for quick assessment of the mental state or assistance in a crisis, but also in positions of comprehensive care for a hospitalized patient [4].

Today, technologies for remote psychiatric care may include communication systems such as mobile phone, the Internet, and email. In general, however, the preferred method of communication is a live video conferencing [5]. It often allows psychiatrists to evaluate both verbal and nonverbal messages, which are part of the analysis during any psychiatric examination. Nevertheless, the popularity of this type of communication with the patient, which is telepsychiatry, has not been too popular, moreover, opinions regarding its effectiveness have been ambiguous [6]. These limitations resulted largely from the aversion of the psychiatrists' community itself, as many critics believed that the lack of personal interaction limited the effectiveness of telepsychiatric therapies [7]. Moreover, many doubts appeared in the formal and legal assessment of this method of conducting medical visits (such as patient identification, verification of personal data and their protection). The lack of financing for these forms of health services in polish conditions was also a significant barrier.
\end{abstract}

Keywords: Telemedicine, Psychiatry, Telepsychiatry, Mental health, Covid-19, Sars-cov2, Pandemic, Stress, Chronic diseases, Lockdown.

\section{Opinion}

The situation related to telemedicine, or more precisely telepsychiatry, changed dramatically in March this year, with the come of the COVID -19 pandemic, which resulted in a lockdown lasting several weeks. This form of contact literally became the only available form of psychiatric care. Based on my own experience of a psychiatrist, who used the communication system mainly by telephone throughout the pandemic period, I can state with full responsibility that the psychiatric health care system survived the worst moments of the pandemic only thanks to the possibility of this kind of communication with the patients. Considering the significant stress that accompanies all of us from the first weeks of the pandemic, and which is particularly burdensome for psychiatric healthcare patients, it should be presumed that telepsychiatry played a significant role in maintaining the stability of the healthcare system-and above all, and perhaps more importantly - in many cases prevented the deterioration of the patients' mental health. 
In a short period of time, the pandemic caused many systemic changes that greatly facilitated the useage of telecommunications, including regulating the financing of this type of medical services. It seems that the rapid introduction and intensive useage of such systems, forced by the situations, also influenced the perception of this method by patients, who did not feel deprived of specialist care. Again, based on my own experience, I can say that a significant part of polish patients was satisfied with this method of communication, some even suggest that they felt more comfortable than during a traditional examination. Perhaps also with the useage of telemedicine, it will be possible to reduce the increasing queues to specialist clinics in the era of the global shortage of medical personnel and to enable faster medical consultations, especially for patients with chronic diseases. The mental health crisis that is likely to emerge as a result of the COVID-19 pandemic and the economic changes it induces is not only an opportunity to develop telepsychiatry and other modern mental health tools, but also a necessity if we are to meet this challenge and help those patients who will especially need it.

\section{Conclusion}

The Covid-19 pandemic brought about significant limitations in interpersonal contacts, it also caused great difficulties in the casual functioning of medical care facilities, including outpatient treatment. Telemedicine, together with the recently introduced possibility of using electronic prescriptions, made it possible to continue psychiatric and psychological therapy to a much greater extent than is currently done in other medical specialties. There is no doubt, however, that online contact will never replace personal contact in the doctor-patient relationship but can become helpful in situations where a direct meeting is not possible.

\section{References}

1. Krzystanek M, Stolarczyk A, Borkowski M, Murawiec S (2018) Telemedicine cognitive behavioral therapy. Psychiatria 15(2): 110-116.

2. Kirsh S, Su GL, Sales A, Jain R (2015) Access to outpatient specialty care: solutions from an integrated health care system. Am J Med Qual 30(1): 88-90.

3. Cipolat C, Geiges M (2003) The History of Telemedicine. In: Burg G Telemedicine and Teledermatology. Curr Probl Dermatol. Basel 32: 6-11.

4. Krzystanek M, Krysta K, Skałacka K (2017) Treatment Compliance in the Long-Term Paranoid Schizophrenia Telemedicine Study. J Technol Behav Sci 2(2): 84-87.

5. Shahrzad M, Ghaffari Nejad A, Mofakhami O, Raaii F, Bahaadi BK (2019) Evaluating the Diagnostic Agreement between Telepsychiatry Assessment and Face-to-Face Visit: A Preliminary Study. Iran J Psychiatry 14(3): 236-241.

6. Salisbury C, O'Cathain A, Edwards L, Thomas C, Gaunt D, et al. (2016) Effectiveness of an integrated telehealth service for patients with depression: a pragmatic randomised controlled trial of a complex intervention. The Lancet 3(6): 515-525.

7. Crawford A, Sunderji N, López J, Soklaridis S (2016) Defining competencies for the practice of telepsychiatry through an assessment of resident learning needs. BMC Med Educ 16: 28. 